\title{
Drenaje urbano sostenible: una alternativa para Xalapa, Veracruz, México
}

\author{
Sustainable Urban Drainage: \\ An Alternative for Xalapa, Veracruz, Mexico
}

Paulo César Parada-Molina, ${ }^{1}$ Ana Isabel Suárez Guerrero, ${ }^{2}$ Joaquín

Jiménez Huerta, ${ }^{3}$ Juan Cervantes Pérez $z^{4}$ y Lily Ariadna Silva Blanco ${ }^{5}$

\section{Resumen}

Las áreas urbanas generan más escorrentía de aguas pluviales que las áreas naturales, debido a que la impermeabilización de las superficies reduce la infiltración del agua. Ante esto, la tendencia de gestión sostenible de recursos naturales ha generado nuevos enfoques de diseño y planeación urbana que incluyen el uso de sistemas de drenaje innovadores. Por ello, el presente estudio tiene como objetivo determinar la viabilidad para la creación e implementación de los pozos de infiltración como sistema de drenaje pluvial sostenible, a nivel vivienda, en zonas urbanas del municipio de Xalapa, Veracruz, México. Los resultados de viabilidad indican que el interés de las personas en adoptar e invertir en infraestructura para el drenaje pluvial (66.67\%), su bajo costo (75\%), así como las características físicas de las zonas (83\% en zonas estables), juegan un papel muy importante en la aplicación de este tipo de proyectos, sin dejar de lado los beneficios ambientales (91.67\%). Las características técnicas (tipo de suelo, geotecnia, capacidad de infiltración) son prioritarias para el uso de drenaje pluvial alternativo en viviendas de Xalapa. Con ello, la construcción y uso de sistemas de drenaje pluvial alternativo es una medida aplicable a las

\footnotetext{
${ }^{1}$ Maestría en Gestión Ambiental para la Sustentabilidad por la Universidad Veracruzana, México. Profesor de la Universidad Veracruzana. Líneas de interés: gestión ambiental, sustentabilidad y gestión de los recursos hídricos. ORCID: https://orcid.org/0000-0001-9567-7142. Correo electrónico: pparada@uv.mx

${ }^{2}$ Doctorado en Ecología y Manejo de Recursos Naturales por el Instituto de Ecología A.C., México. Profesor de la Universidad Veracruzana. Líneas de interés: ambiente, ecología del ecosistema y manejo de recursos naturales. ORCID: https://orcid.org/0000-0002-0864-2230. Correo electrónico: asuarez@uv.mx

${ }^{3}$ Maestría en Desarrollo Rural Sustentable por el Colegio de Postgraduados, México. Profesor de la Universidad Veracruzana. Líneas de interés: gestión ambiental, sustentabilidad y desarrollo rural. ORCID: https://orcid.org/0000-0003-3705-9500. Correo electrónico: jojimenez@uv.mx

${ }^{4}$ Autor de correspondencia. Doctorado en Geografía por la Universidad Nacional Autónoma de México, México. Profesor de la Universidad Veracruzana. Líneas de interés: cambio climático, impactos ambientales locales y regionales, hidrología y bioclimatología. ORCID: https://orcid.org/0000-0002-1429-3348. Correo electrónico: jcervantes@uv.mx

5 Doctorado en Investigación Educativa por la Universidad Veracruzana, México. Profesor de la Universidad Veracruzana. Líneas de interés: educación, sustentabilidad y desarrollo. ORCID: https://orcid.org/0000-0001-8450-9784. Correo electrónico: lysilva@uv.mx
} 
viviendas del área urbana de Xalapa, por lo que pueden ser un complemento del sistema de drenaje tradicional.

Palabras clave: desarrollo urbano; drenaje pluvial; viabilidad; pozos de infiltración; sostenibilidad;

\begin{abstract}
Urban areas generate more stormwater runoff than natural areas because waterproofing of surfaces reduces water infiltration. Given this, the trend of sustainable management of natural resources has created new approaches to urban design and planning that include the use of innovative drainage systems. Therefore, the present study aims to determine the viability for the creation and implementation of infiltration wells as a sustainable storm drainage system, at the household level, in urban areas of the municipality of Xalapa, Veracruz, Mexico. The viability results indicate that people's interest in adopting and investing in infrastructure for storm drainage $(66.67 \%)$, its low cost (75\%), as well as the physical characteristics of the areas (83\% in stable areas) are relevant in the application of this type of project, without neglecting the environmental benefits (91.67\%). The technical characteristics (the soil, geotechnics, infiltration capacity) are a priority for the use of alternative storm drainage in houses in Xalapa. With this, the construction and use of alternative storm drainage systems are a measure applicable to houses in the urban area of Xalapa, which can be a complement to the traditional drainage system.
\end{abstract}

Keywords: urban development; storm drain; viability; infiltration wells; sustainability;

\title{
Introducción
}

El proceso de urbanización incrementa las escorrentías de aguas pluviales debido a que se reemplazan la superficie del suelo y la vegetación con áreas impermeables, lo que reduce la infiltración de agua en el suelo y su almacenamiento en zonas subterráneas (Jaume, 2016). Un ejemplo es el caso de Xalapa, Veracruz, México, que en los últimos 40 años ha incrementado $41.5 \mathrm{~km}^{2}$ su superficie urbana (Lemoine, 2012).

De acuerdo con el Banco Mundial, el crecimiento urbano abrupto sobrepasa cualquier previsión, diseño y planeación de servicios urbanos. 
Particularmente, la poca planeación de prácticas de gestión del agua ha incrementado los problemas de escasez, contaminación de cuerpos de agua, aumento de los caudales máximos y los volúmenes de agua superficiales, siendo cada vez más frecuente observar problemas de encharcamientos, inundaciones y sedimentación en zonas urbanas; erosión en los sectores más vulnerables; degradación y pérdida de arroyos superficiales y subterráneos (Chang et al., 2009; Moguel, 2011; Walsh et al., 2012; Sánchez-Rodríguez y Cavazos, 2015; Romero-Guzmán et al., 2018). Esto ha generado la necesidad de implementar una red de drenaje centralizado para canalizar la escorrentía directamente a los cuerpos de agua (Fanelli et al., 2017). Comúnmente, dicha red presenta problemas de obstrucción que favorece encharcamientos e inundaciones que afectan zonas altamente pobladas (Audefroy y Padilla, 2018; Rojas et al., 2019), además de perder beneficios, como la reducción de la contaminación del agua por arrastre, más allá de la atenuación de los escurrimientos superficiales (Stovin et al., 2013).

Ante ello, destaca la necesidad de aplicar principios del desarrollo sustentable al diseño urbano (Mulder, 2007; Kogan y Bondorevsky, 2016). Las llamadas "técnicas alternativas de desarrollo urbano sostenible" pueden ayudar a resolver dichos problemas a partir de Buenas Prácticas de Gestión del Agua Pluvial (BPAs, por sus siglas en inglés) o Sistemas Urbanos de Drenaje Sostenible (SUDS) que, a diferencia de las técnicas tradicionales, tienen como objetivo minimizar los problemas asociados al ciclo del agua en áreas urbanas, disminuyendo el impacto del agua pluvial, ya que ésta es tratada en el lugar donde se genera, almacenando y/o infiltrando el escurrimiento superficial proveniente de los techos de las viviendas (Azzout et al., 1994; Perales-Momparler y Andrés-Doménech, 2008; Ashley et al., 2013; Woods-Ballard et al., 2015; Bouarafa et al., 2019). Los sistemas alternativos de drenaje pluvial son una solución eficaz para el tratamiento en origen de las escorrentías, aún en terrenos prácticamente impermeables, ya que de acuerdo con García (2011) y Perales-Momparler et al. (2014) se puede controlar más del $90 \%$ de los eventos de lluvia y de los volúmenes de escorrentía generados.

Las prácticas de manejo de aguas pluviales basadas en infiltración, como las zanjas y pozos de infiltración, pavimento permeable, y techos verdes (VanWoert et al., 2005; Qin et al., 2013), han demostrado el potencial para reducir la escorrentía y mejorar la calidad del agua. Un pozo de infiltración se utiliza para drenar agua a través de sus paredes y del fondo hacia cuerpos de agua subterráneas. Éstos pueden estar rellenos de agregados pétreos o estar completamente huecos en su interior, permitiendo almacenar temporalmente el agua de lluvia e infiltrarla posteriormente de forma directa hacia el suelo a través de sus paredes y el fondo (Figura 1). 
Figura 1. Esquema de pozos de infiltración, pozo hueco y pozo relleno de agregados pétreos
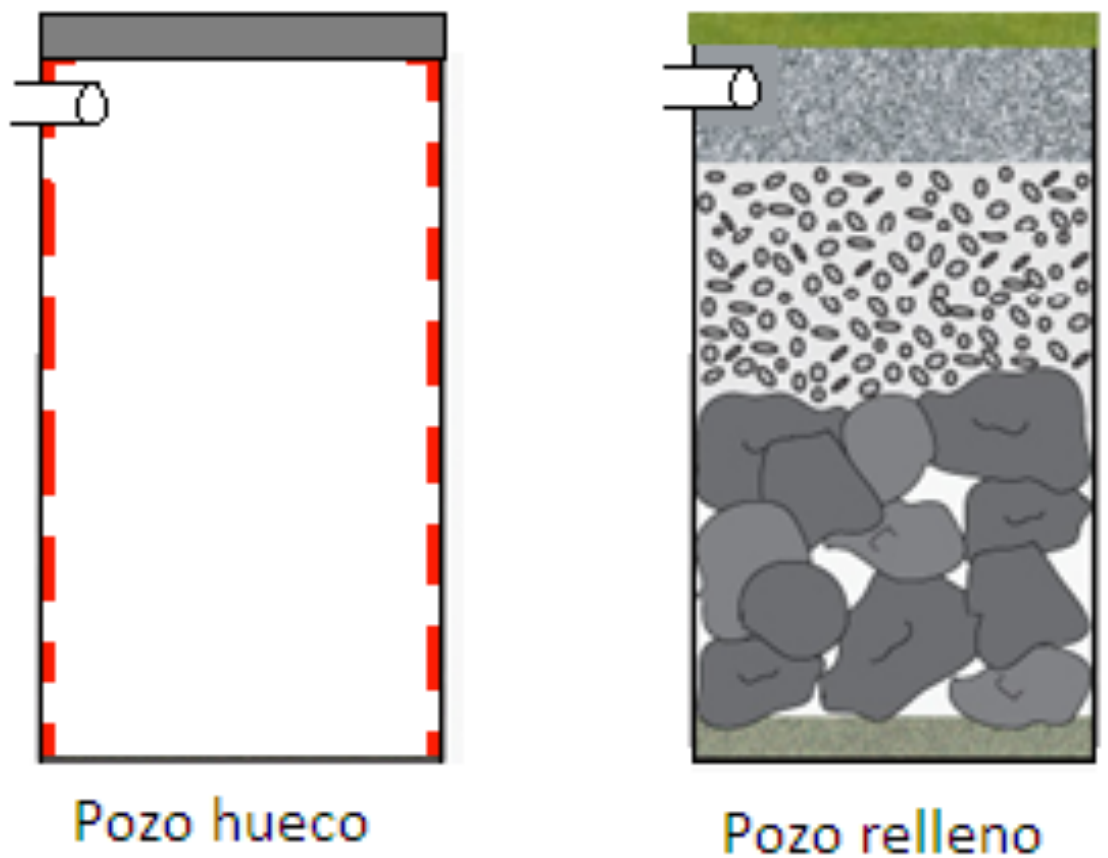

Nota: El pozo relleno puede tener un jardín (franja verde del esquema) en su superficie.

Fuente: elaboración propia.

El uso de pozos de infiltración es conveniente en zonas donde no hay mucho espacio disponible para obras de mayor tamaño, pero existe capacidad de infiltración del suelo (Woods-Ballard et al., 2015). Estos pozos son utilizados para drenar superficies pequeñas que no reciben grandes cantidades de contaminantes, como es el caso de la azotea de una casa. Además, por su diseño, en los pozos con relleno se pueden adaptar jardines en su superficie (Autixier et al., 2014; Katsifarakis et al., 2015).

Por otra parte, Walsh et al. (2014), encontraron que el escurrimiento superficial de agua, generado en los eventos de lluvia, se puede reducir hasta en un $15 \%$ por el agua que es retenida y almacenada en pozos y cisternas de las viviendas de la cuenca Chollas Creek, San Diego, California, Estados Unidos, mientras que Autixier et al. (2014) encontraron valores de hasta 20 \% de reducción con el uso de áreas de infiltración. También, el uso de zanjas filtrantes ha favorecido la reducción de flujos superficiales de agua ocasionados por las lluvias intensas en Chesapeake Bay, Estados Unidos (Loperfido et al., 2014).

En México, son pocos los estudios encontrados sobre el uso de pozos de infiltración a nivel vivienda. Desde 2009, de acuerdo con el Reglamento de Zonificación Urbana del Municipio de Guadalajara, es de carácter obligatorio 
que las viviendas cuenten con este tipo de sistemas para canalizar el agua de lluvia, como estrategia para favorecer la recarga de los cuerpos de agua de esa zona. Se han realizado propuestas para el uso de pozos de infiltración en el diseño de modelos de vivienda sustentable para áreas urbanas (Gutiérrez, 2017) y rurales (Peña, 2014), así como el uso de pavimentos permeables (Lizárraga-Mendiola et al., 2015). Para el municipio de Xalapa, México, no se han encontrado registros sobre el uso de estos sistemas de drenaje, por lo que es necesario plantearse las siguientes preguntas: ¿Es viable la integración de pozos de infiltración como sistema de drenaje pluvial en viviendas del municipio de Xalapa? ¿Existe conocimiento e interés de las personas en temas relacionados con el drenaje de agua pluvial? ¿Cuáles son las condiciones para la implementación de pozos de infiltración y su buen funcionamiento en viviendas de Xalapa? ¿Qué impactos ambientales se pueden generar o mitigar a partir de la construcción de los pozos de infiltración superficial?

Debido a lo anterior, el objetivo de este trabajo fue determinar la viabilidad para la creación e implementación de los pozos de infiltración como sistema de drenaje pluvial alternativo, a nivel vivienda, en zonas urbanas del municipio de Xalapa. Con esto, se podrá contar con las bases de diseño de sistemas alternativos de drenaje pluvial de bajo costo, que pueda ser adoptado por la sociedad para mitigar algunas afectaciones al ambiente y a la población.

\section{Materiales y métodos}

\section{$\underline{\text { Zona de estudio }}$}

El municipio de Xalapa, Veracruz, México, se ubica entre los $19^{\circ} 30^{\prime}$ y $19^{\circ} 36^{\prime}$ de latitud norte y $96^{\circ} 49^{\prime}$ y $96^{\circ} 59^{\prime}$ de longitud oeste, a una altitud de 1300 a 1500 metros sobre el nivel del mar (Figura 2). Tiene una superficie de 124.38 $\mathrm{km}^{2}$ que representa el $0.16 \%$ de la superficie del estado de Veracruz. Bajo el sistema de clasificación climática de Köppen modificada por García (2004), Xalapa tiene un clima semicálido húmedo con lluvias distribuidas todo el año ((A) $\mathrm{Cb}(\mathrm{fm})$ ), temperatura media anual de $19.2^{\circ} \mathrm{C}$, y un promedio anual de precipitación pluvial de $1435.8 \mathrm{~mm}$, siendo los meses más lluviosos julio y septiembre (Normales climatológicas 1981-2010, SMN). En función del tipo y la geotecnia del suelo, Huesca (2009) clasifica el suelo de Xalapa en cuatro tipos: 1) suelo estable; 2) suelo medianamente estable; 3) suelo blando, y 4) suelo muy blando. 
Figura 2. Ubicación del municipio de Xalapa, Veracruz

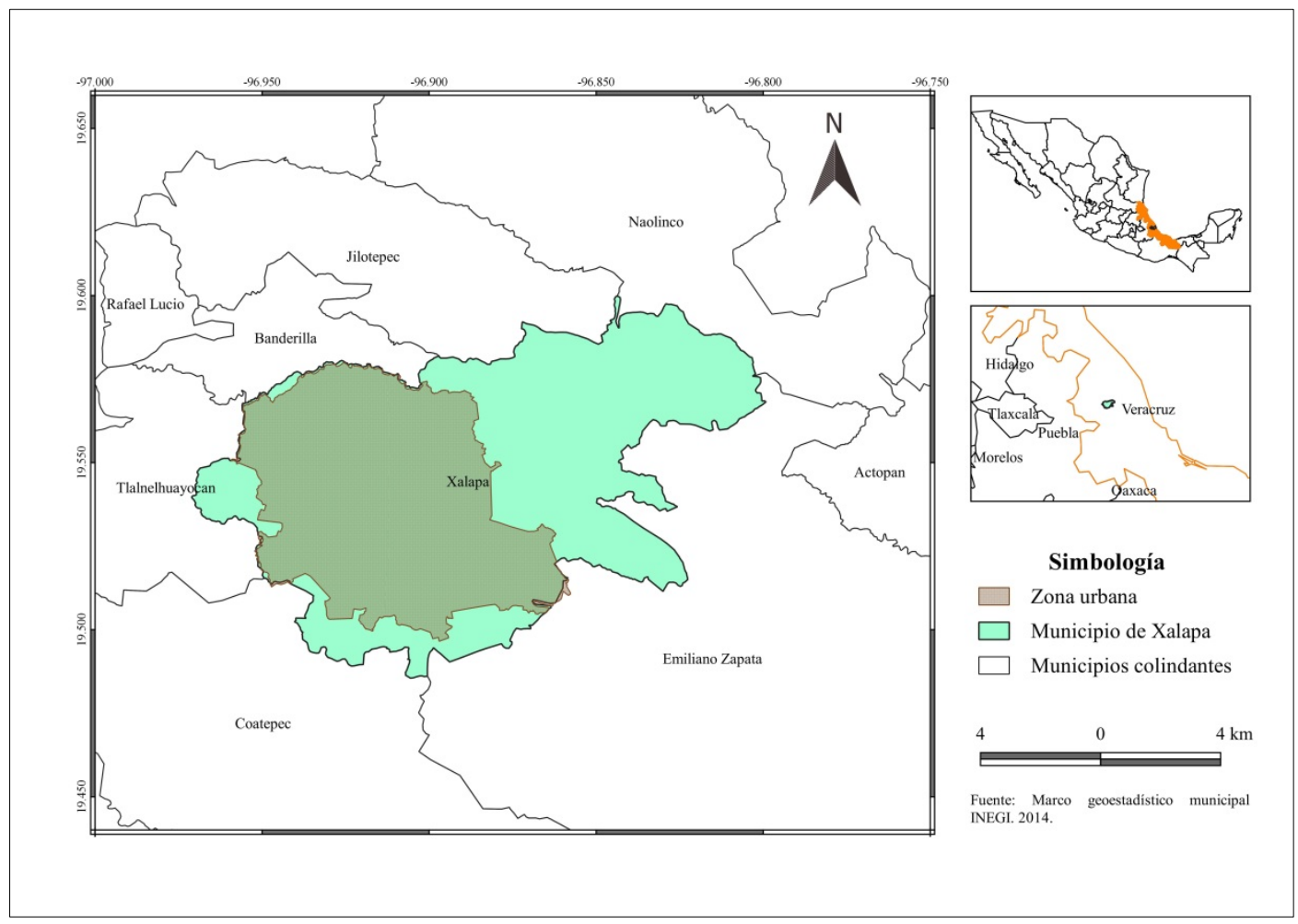

Fuente: elaboración propia a partir del marco geoestadístico municipal (INEGI, 2014).

\section{Estudio de viabilidad}

La viabilidad se determinó con análisis particulares de aspectos sociales, económicos, técnicos y ambientales, que influyen o podrían influir en el proyecto para la implementación de los pozos de infiltración superficial como sistemas de drenaje pluvial en el área de estudio.

\section{Viabilidad social y económica}

Con la finalidad de conocer la percepción y el interés de las personas en temas relacionados con el drenaje de agua pluvial, se llevó a cabo una encuesta. Para ello se seleccionaron a 384 personas distribuidas equitativamente y de manera aleatoria, considerando un nivel de confianza del 95 \% (Spiegel y Stephens, 2009), en tres colonias del municipio de Xalapa: Carolino Anaya, Progreso Macuiltépetl y Héroes Ferrocarrileros, localizadas en una misma subcuenca urbana. La encuesta se basó en siete preguntas sobre aspectos como caracterización social (colonia, edad, sexo, escolaridad), hábitos de aprovechamiento y/o manejo del agua pluvial, conocimiento de sistemas de manejo del agua pluvial, e interés de invertir para implementarlos en sus viviendas. La población objetivo estuvo en un 
intervalo de edad de entre 25 y 55 años, la cual se consideró que tenía capacidad de decisión y adquisición en cuanto a economía del hogar se refiere. Para la parte económica, se estableció el costo unitario de la construcción de los pozos de infiltración empleando el software OPUS, y se relacionó con la información referente a la disposición e interés de inversión en infraestructura para el manejo del agua pluvial.

\section{Viabilidad técnica}

Se determinaron los parámetros de construcción de los pozos de infiltración para cada colonia; el volumen, $\mathrm{V}$, fue calculado con la ecuación 1:

$$
\mathrm{V}=\left(720000 \mathrm{QM}_{\mathrm{M}} / \mathrm{K}_{\mathrm{if}}\right)^{3 / 2} \quad \text { Ec. } 1
$$

donde, $\mathrm{K}_{\text {if }}$ es la velocidad de infiltración del agua por el fondo del pozo y la sección de las paredes (m/d), y Qm es el caudal máximo de agua que fluirá a través de una azotea hacia el pozo de infiltración (CIDECALLI, 2001).

La velocidad de infiltración se calculó a partir de ocho muestreos por colonia, empleando el método Porchet (Imbe et al., 1995; MINVU-PUC, 1996) (Ec. 2);

$$
K_{i f}=\frac{R}{2\left(t_{1}-t_{2}\right)} \operatorname{Ln}\left(\frac{2 H_{1}+R}{2 H_{2}+R}\right) \quad \text { Ec. } 2
$$

donde, $\mathrm{R}$ es el radio del pozo $(\mathrm{mm}), \mathrm{H}_{1}$ y $\mathrm{H}_{2}$ son las alturas (mm) de agua medidos en los instantes $\mathrm{t}_{1} \mathrm{y} \mathrm{t}_{2}(\mathrm{hr})$, respectivamente.

El caudal máximo de agua $\left(\mathrm{Q}_{\mathrm{M}}\right)$ se determinó a partir de la ecuación 3, propuesta por CIDECALLI (2001):

$$
Q_{M}=0.2778 C_{e} I A_{C} \quad \text { Ec. } 3
$$

donde, Ce es el coeficiente de escurrimiento y $A_{c}$ es el área de captación (área de la azotea) en $\mathrm{km}^{2}$.

La intensidad de la lluvia máxima de diseño (I) se calculó a partir de los datos de precipitación máxima diaria correspondientes al periodo 1961-2011 
del observatorio meteorológico de la ciudad de Xalapa, Veracruz, con la ecuación propuesta por Pereyra-Díaz et al. (2005) (Ec. 4):

$$
I_{t}^{T}=\frac{2257.52 \log \left(10^{(0.72)} \mathrm{T}^{(0.28)}\right)}{(t+9.9)^{0.82}} \quad \text { Ec. } 4
$$

donde, $\mathrm{I}_{\mathrm{t}}^{\mathrm{T}}$ es la intensidad promedio de la lluvia para cualquier duración $\mathrm{t}$ (minutos) y periodo de retorno $\mathrm{T}$ (años). Considerando las características de las colonias en las que se realizó este trabajo, se tomó el periodo de retorno de 1.5 años, por tratarse de "Vialidad local-avenidas y calles" cuya importancia no traspasa la zona servida (CONAGUA, 2019). Teniendo los parámetros de construcción, se realizó el diseño de los pozos de infiltración.

Para concretar la instalación de prototipos en viviendas de las colonias estudiadas, se estableció comunicación con ocho jefes de manzana de cada colonia, y se llevaron a cabo cuatro pláticas de divulgación de temas relacionados con la importancia del manejo y uso del agua pluvial, estrategias de manejo del agua de lluvia, y del diseño y construcción de los pozos de infiltración superficial a nivel vivienda como sistema de drenaje alternativo. Posteriormente se les invitó a instalar un prototipo en su vivienda.

Para verificar el funcionamiento de los pozos en las viviendas, se dio seguimiento durante un año, a través de un formato de registro de las condiciones de los pozos de infiltración durante eventos de lluvia. El registro incluía la fecha con evento de lluvia, el estado del pozo de infiltración durante el evento: 1) húmedo, 2) muy húmedo, 3) brota agua, o 4) escurre agua con tierra; y comentarios generales. Esta información se comparó con los datos diarios de lluvia emitidos por la Comisión Nacional del Agua (CONAGUA) y se relacionó con el funcionamiento de los pozos de infiltración.

\section{Viabilidad ambiental}

Se realizó una revisión documental para identificar los impactos ambientales que se pueden generar o mitigar a partir de la construcción de los pozos de infiltración superficial a nivel vivienda como sistema de drenaje pluvial alternativo. La información recabada se relacionó con los datos obtenidos a partir de visitas a las viviendas de manera mensual para monitorear el funcionamiento y las condiciones físicas de los pozos de infiltración, así como las actividades de mantenimiento y limpieza reportadas por los participantes. 


\section{Análisis de la viabilidad}

Se identificaron indicadores considerados como prioritarios en cada una de las componentes diagnosticadas (social, técnica, económica y ambiental), para determinar si el proyecto era viable en cada una de ellas. Con la finalidad de darle mayor peso a las componentes técnica y social, por considerarse los componentes de mayor importancia para la realización de este proyecto, se seleccionaron seis indicadores técnicos y seis sociales, así como tres relacionados al costo de inversión y tres ambientales, ya que si no se cuentan con las características idóneas y la sociedad no está en disposición de participar, los proyectos a esta escala suelen no prosperar (Massolo, 2015). A cada indicador considerado como prioritario se le asignó un valor de 0 a 4 (Cuadro 1), tomando como referencia la metodología propuesta por Pacheco et al. (2011), y se realizó la suma para obtener el puntaje total de cada componente.

Cuadro 1. Valoración de los indicadores prioritarios

\begin{tabular}{|l|c|}
\hline \multicolumn{1}{|c|}{ Descripción } & Valor \\
\hline Si el indicador no se cumple & 0 \\
\hline Si el indicador se cumple escasamente & 1 \\
\hline Si el indicador se cumple medianamente & 3 \\
\hline Si el indicador se cumple & 4 \\
\hline Fuente: elaboración propia. &
\end{tabular}

Los puntajes totales obtenidos se relacionaron con los puntajes óptimos para determinar la ponderación total del proyecto, y la ponderación óptima, con las ecuaciones 5 y 6 (Pacheco et al., 2011).

$$
\begin{gathered}
\text { Ponderación óptima }=100 * \frac{\text { Puntaje óptimo de cada componente }}{\text { Puntaje total óptimo }} \text { Ec. } 5 \\
\text { Ponderación obtenida }=100 * \frac{\text { Puntaje obtenido de cada componente }}{\text { Puntaje total óptimo }} \text { Ec. } 6
\end{gathered}
$$

Para obtener la viabilidad social, técnica, económica y ambiental se relacionó la ponderación óptima y la ponderación obtenida en cada componente analizado (ecuación 7): 
Porcentaje de viabilidad $=100 * \frac{\text { Porcentaje obtenido }}{\text { Porcentaje óptimo }}$

Ec. 7

Para la interpretación de la viabilidad de este proyecto se diseñaron los siguientes criterios:

- Viable: el proyecto cumple con las características necesarias para su operación, se puede ejecutar y obtener el impacto previsto; es decir, cuando sus cuatro componentes y la ponderación total tienen porcentajes mayores al $66.1 \%$.

- Medianamente viable: se deben tomar ciertas medidas correctivas para que el proyecto pueda ser ejecutado en un futuro; es decir, está evaluado con una ponderación total entre el $33.1 \%$ al $66 \%$ o sólo tres de sus componentes cuentan con porcentajes de viabilidad mayores al $66 \%$.

- No viable: el proyecto no cuenta con las características necesarias para su funcionamiento; es decir, que está evaluado con una ponderación total menor al $33.1 \%$ o sólo dos de sus componentes cuentan con porcentajes de viabilidad mayores al $66 \%$.

\section{Resultados y discusión}

\section{Percepción e interés social}

El 94 \% de la población encuestada, mencionó contar con sistema de drenaje. Algunos encuestados puntualizaron que este servicio lo tienen desde hace no más de cinco o seis años en la colonia Carolino Anaya, y no más de 10 años en algunas zonas de la colonia Progreso Macuiltépetl; en la colonia Héroes Ferrocarrileros el sistema de drenaje es más antiguo. El $4 \%$ restante usa fosas sépticas como sistemas de desagüe, ya que en las tres colonias existen "resumideros" que son utilizados como sistemas de drenaje. Referente al manejo del agua pluvial, $59.9 \%$ de las personas encuestadas comentaron que desechan el agua directamente a las calles; el $29.7 \%$ la desechan a través del drenaje; y el $10.4 \%$ realizan actividades de almacenamiento de agua u otro uso como lavar los patios.

El $100 \%$ de los encuestados mostró interés en adoptar una infraestructura para el manejo del agua pluvial, sin embargo, el $73.7 \%$ está dispuesto a invertir una cantidad menor o igual a $\$ 1000.00$ (un mil pesos 00/100 M.N.), y el $26.3 \%$ restante a invertir una cantidad mayor. De acuerdo con Papafotiou y Katsifarakis (2015), el conocimiento escaso o nulo de estos 
sistemas y la poca difusión de sus ventajas, ha influido en el desarrollo de construcciones tradicionales donde el servicio de drenaje es provisto por dependencias municipales, y por lo tanto, en que la población vea como un gasto invertir en otra infraestructura, y considere innecesario realizar actividades relacionadas con el manejo y aprovechamiento del agua pluvial. Por ello, es importante crear y proponer estrategias económicas atrayentes, que puedan ser implementadas para el beneficio de la población.

\section{Diseño y funcionamiento}

Con base en la velocidad de infiltración $\left(\mathrm{K}_{\mathrm{if}}\right)$ en las tres colonias, se ha encontrado que cada una cuenta con características particulares, por lo que las obras e infraestructura deberán adaptarse a dichas condiciones. Los valores de velocidad de infiltración fueron mayores en las colonias Carolino Anaya y Progreso Macuiltépetl (Cuadro 2). La compactación del suelo a la escala del lote urbano es un proceso que reduce las tasas de infiltración (Jaume, 2016). Esto se presentó de manera más evidente en la colonia Héroes Ferrocarrileros donde la tasa de infiltración fue relativamente baja $(68 \mathrm{~mm} / \mathrm{h})$.

\section{Cuadro 2. Velocidad de infiltración promedio para cada colonia del municipio de Xalapa}

\begin{tabular}{|l|c|c|}
\hline \multicolumn{1}{|c|}{ Colonia } & $\mathbf{K}_{\mathrm{if}}\left(\mathbf{X 1 0} \mathbf{- 5}^{-5} \mathbf{m} / \mathbf{s}\right)$ & $\mathbf{K}_{\mathrm{if}}(\mathbf{m m} / \mathbf{h})$ \\
\hline Carolino Anaya & 4.036 & 145.3 \\
\hline Héroes Ferrocarrileros & 1.905 & 68.8 \\
\hline Progreso Macuiltépetl & 6.405 & 230.6 \\
\hline
\end{tabular}

Fuente: elaboración propia.

Se determinó que la lluvia máxima en 1 hora, calculada para el periodo de retorno de 1.5 años, es de $74.1 \mathrm{~mm}$, el coeficiente de escurrimiento (Ce) del concreto, de acuerdo con Campos-Aranda (2010), es de 0.75 y el área de captación propuesta fue de $30 \mathrm{~m}^{2}$. A partir de estos parámetros se calculó un caudal máximo de agua de $0.00044 \mathrm{~m}^{3} / \mathrm{s}$, a partir del cual se diseñaron los pozos de infiltración para viviendas.

Con estos parámetros se determinó que el volumen de diseño de los pozos de infiltración (Figura 3) debe ser de $2.744 \mathrm{~m}^{3}$ y $1.33 \mathrm{~m}^{3}$ para las colonias Carolino Anaya y Progreso Macuiltépetl, respectivamente (Cuadro 3). El volumen de diseño para la colonia Héroes Ferrocarrileros limitó la implementación de pozos de infiltración a nivel vivienda debido a que fue de gran magnitud $\left(9.26 \mathrm{~m}^{3}\right)$. Por tal motivo, no se encontraron personas 
interesadas en la infraestructura propuesta; ante ello, no se instalaron prototipos en esa colonia. En las dos colonias restantes, se concretó la participación de ocho personas, por lo que se instalaron cuatro prototipos en cada una de ellas (Figura 4).

\section{Cuadro 3. Volumen mínimo requerido para los pozos de infiltración} para tres colonias del municipio de Xalapa, Veracruz

\begin{tabular}{|l|c|}
\hline \multicolumn{1}{|c|}{ Colonia } & Volumen $\left(\mathbf{m}^{\mathbf{3}}\right)$ \\
\hline Carolino Anaya & 2.744 \\
\hline Héroes Ferrocarrileros & 9.261 \\
\hline Progreso Macuiltépetl & 1.331 \\
\hline
\end{tabular}

Fuente: elaboración propia.

Figura 3. Diseño de prototipo de pozo de infiltración relleno de agregados pétreos

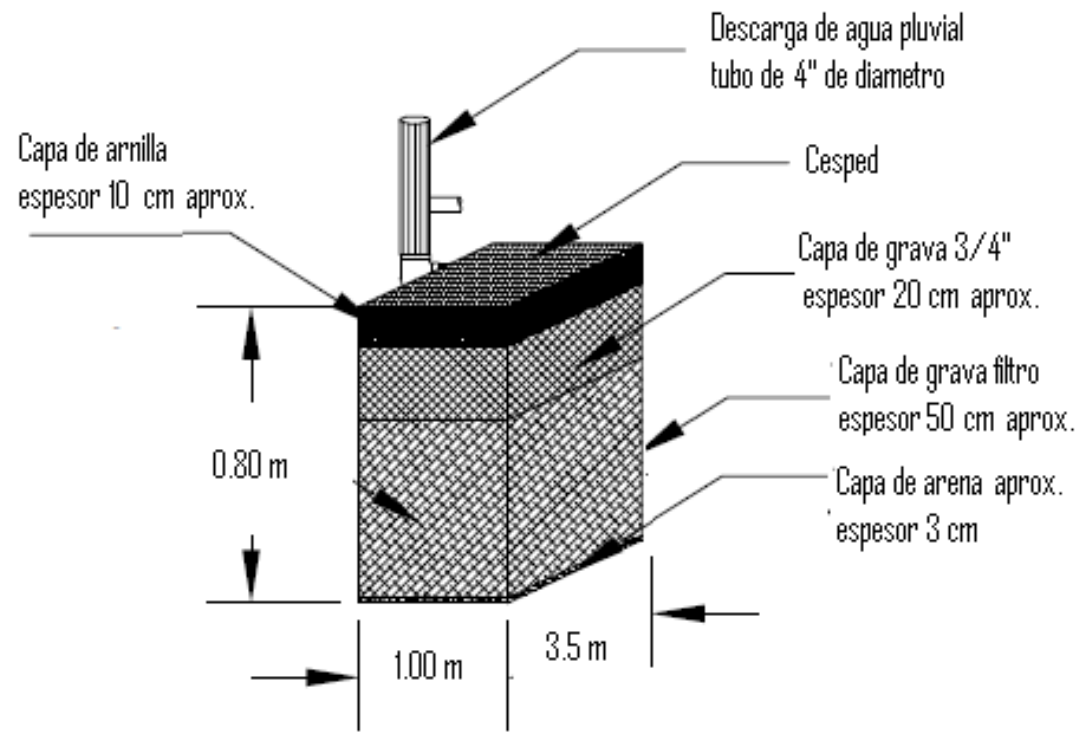

Fuente: elaboración propia. 
Figura 4. Ubicación de los pozos de infiltración en las Colonias Carolino Anaya y Progreso Macuiltépetl, Xalapa, Veracruz

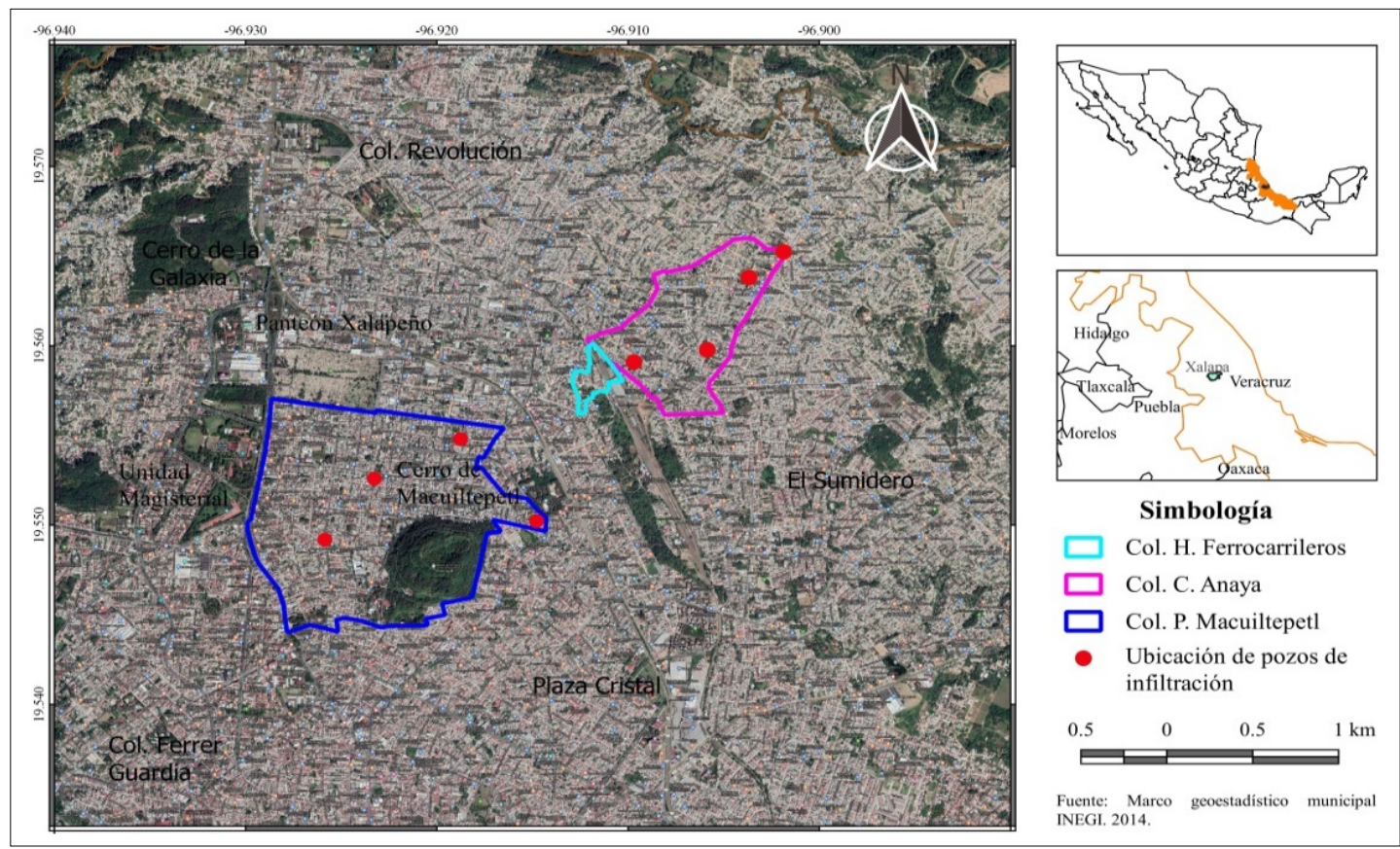

Fuente: elaboración propia a partir del marco geoestadístico municipal (INEGI, 2014).

En una primera etapa del seguimiento, se realizaron visitas mensuales $(n=7)$ a las viviendas, con la finalidad de recabar la información en cada una de ellas a partir de las observaciones realizadas y anotadas por las personas participantes. De acuerdo con el formato de registro, se pudo notar que de los ocho prototipos instalados, uno ubicado en la colonia Progreso Macuiltépetl, emplazado en una jardinera pequeña, presentó afloramiento excesivo de agua, al grado de quedar inundado hasta el nivel de las paredes. Este pozo no se pudo hacer con las dimensiones correspondientes al área de captación (la azotea) debido al tamaño reducido de la jardinera. En los siete prototipos restantes, el funcionamiento fue adecuado para contener el escurrimiento generado por lluvias menores a $74 \mathrm{~mm}$.

Por otro lado, se observó en los formatos de registro, y en comentarios de las personas, que hubo días en que las lluvias fueron muy abundantes, lo que provocó que el agua escurriera o aflorara en exceso de los pozos instalados. Debido a que los pozos de infiltración instalados están diseñados para contener e infiltrar cierta cantidad de agua, se hizo hincapié en la importancia de colocar una salida alterna de agua o desagüe para desechar el exceso, y con ello evitar la erosión y sobresaturación del suelo, y que el césped se pudriera por la gran cantidad de agua. Papafotiou y Katsifarakis (2015) mencionan que el buen funcionamiento de la infraestructura de drenaje pluvial sostenible depende de la velocidad de infiltración de agua en el suelo donde se pretenden implementar, por ello es necesario diseñar los 
pozos de infiltración a partir de cantidad de agua que se pretenda infiltrar y del tipo de sustrato (permeabilidad y porosidad).

En la segunda etapa de visitas mensuales, se recabó la información restante para el análisis del funcionamiento. Con base en la información se pudo notar que los pozos de infiltración continuaron funcionando de manera adecuada para intensidades de lluvia para las que fueron diseñados $(\leq 74$ $\mathrm{mm}$ ); en ese sentido, Autixier et al. (2014) señalan que la infraestructura creada sólo puede contener la cantidad de agua para la que fue diseñada, por lo que el cálculo para su diseño es fundamental.

\section{Costos}

Para determinar el costo de construcción de los pozos de infiltración, se empleó el software OPUS, que es utilizado para la elaboración de presupuestos de obra en el área de la construcción, basándose en el costo de los materiales, la cantidad del material requerido y el costo de mano de obra en la zona. El presupuesto de construcción incluyendo materiales y mano de obra de estos pozos de infiltración fue de $\$ 1153.38$ (un mil ciento cincuenta y tres pesos 38/100 M.N.) antes de impuestos; que si bien no es la cantidad que las personas están dispuestas a invertir, se considera que tiene un bajo costo, lo que puede ser un punto importante para la adopción de este tipo de sistemas de infiltración para el drenaje de agua pluvial a nivel vivienda. Las instalaciones hidráulicas convencionales que consisten en una rápida evacuación de aguas pluviales hacia entornos acuáticos naturales a través de sistemas de alcantarillado, sólo consideran los problemas de gestión de la cantidad de agua, no obstante, son económica y ecológicamente costosos (Bouarafa et al., 2019).

La infraestructura de drenaje pluvial sostenible es económica (Papafotiou y Katsifarakis, 2015). Al determinar el costo de diversas prácticas de manejo de agua pluvial, Joksimovic y Alam (2014) indicaron que los pozos de infiltración como sistemas de drenaje pluvial son de los más económicos. La aplicación de estos sistemas podría incrementarse si las autoridades regulan el desarrollo urbano y la gestión del agua (suministro y drenaje) de manera más eficiente (Brito-Castillo y Pedrozo-Acuña, 2015), e implementan incentivos para promover la participación del sector social y privado en los proyectos de gestión de drenaje sostenible. Estos incentivos podrían incluir apoyo económico para el mantenimiento de estos sistemas y la reducción en las tarifas de suministro de agua y alcantarillado, entre otros (Ando y Freitas, 2011; Valderrama y Levine, 2012). 
Paulo César Parada-Molina, Ana Isabel Suárez Guerrero,

\section{$\underline{\text { Impactos ambientales }}$}

Por el buen funcionamiento de los pozos de infiltración se podría elucidar sobre posibles impactos positivos, como la reducción de los escurrimientos superficiales en las zonas urbanas de Xalapa, la disminución de inundaciones y de encharcamientos, ya que su uso generalizado reduciría alrededor del $25 \%$ del agua que escurre por las calles de Xalapa (5.7 millones de $\mathrm{m}^{3}$ al año, aproximadamente). Autixier et al. (2014) aluden que la reducción de estas problemáticas dependerá del porcentaje de área impermeable que sea drenada hacia los dispositivos de infiltración. Otro impacto positivo sería la recuperación de áreas verdes urbanas, ya que de las ocho viviendas donde se instalaron los prototipos, sólo dos contaban con jardines, por lo que, de implementarse de manera generalizada, se incrementarían las áreas con vegetación en la ciudad. Así, este tipo de infraestructura permite incrementar el diseño estético de las zonas urbanas (Papafotiou y Katsifarakis, 2015; OssaMoreno et al., 2017), aparte de mejorar la calidad del espacio urbano y permitir el manejo del agua pluvial, al favorecer la infiltración y retención de agua en el suelo (Suárez et al., 2008).

En cuanto a impactos negativos, se identificó la posible contaminación, local y difusa, del suelo y del agua subterránea cuando el agua vertida en el pozo de absorción contiene residuos sólidos o impurezas (Rodríguez-Eugenio et al., 2019) por la falta de limpieza de la azotea y la presencia de basura sobre el césped del pozo. Además, las personas de las viviendas observaron un incremento de excretas de perros en el césped (superficie del pozo de infiltración). En las primeras visitas, también se pudo notar que la superficie de dos pozos de infiltración era utilizada para el almacenamiento de basura, entre las que destacaban bolsas y botellas de plástico, desechos sanitarios, residuos de comida (cáscaras de frutas, verduras, grasas, y varios más), latas de alimentos procesados, entre otros. Éstos, junto con el efecto del agua, generan lixiviados que pueden ser vertidos de manera natural al suelo y contaminarlo. De acuerdo con Ellis et al. (2016), debido a que la operación y mantenimiento es responsabilidad de los propietarios, también lo es el buen funcionamiento y la minimización de impactos, por lo deben ser informados de los efectos de un mal manejo y mantenimiento de los pozos de infiltración.

Las diversas actividades realizadas en las ciudades producen una gran variedad de contaminantes que se eliminan en el aire y en las superficies; pueden ser orgánicos, como hidrocarburos, aceites y grasas; inorgánicos, tales como metales y nutrientes disueltos; o microorganismos patógenos, como bacterias y virus (Mikkelsen et al., 1996). Ante esto, es importante remarcar que la sociedad desempeña un papel importante en estas problemáticas. La calidad del agua de lluvia depende de las superficies por las que escurre, ya que la basura producida es arrastrada por ésta y termina en los cuerpos de agua receptores (Fanelli et al., 2017); de modo que al evitar que escurra por superficies contaminadas se previene el deterioro de cauces naturales y también se contribuye a la conservación de las reservas de agua subterránea (Serrano, 2014). 


\section{Determinación de la viabilidad}

Se analizaron los puntos de cada uno de los diagnósticos realizados y se identificaron los indicadores sociales, técnicos, económicos y ambientales considerados prioritarios para la implementación de pozos de infiltración como sistemas de drenaje pluvial (Cuadro 4).

Cuadro 4. Indicadores sociales, técnicos, económicos y ambientales para la construcción de pozos de infiltración superficial a nivel vivienda

\begin{tabular}{|c|c|c|c|}
\hline $\begin{array}{c}\text { Indicadores } \\
\text { sociales }\end{array}$ & $\begin{array}{c}\text { Indicadores } \\
\text { técnicos }\end{array}$ & $\begin{array}{l}\text { Indicadores } \\
\text { económicos }\end{array}$ & $\begin{array}{l}\text { Indicadores } \\
\text { ambientales }\end{array}$ \\
\hline $\begin{array}{l}\text { Personas afectadas por } \\
\text { encharcamientos o } \\
\text { inundaciones }\end{array}$ & Tipo de suelo andosol & \multirow[t]{2}{*}{ Monto dispuesto a invertir } & \multirow{2}{*}{$\begin{array}{l}\text { Reducción de } \\
\text { encharcamientos o } \\
\text { inundaciones }\end{array}$} \\
\hline $\begin{array}{l}\text { Prácticas de manejo de agua } \\
\text { pluvial }\end{array}$ & $\begin{array}{c}\text { Característica geotécnica } \\
\text { estable }\end{array}$ & & \\
\hline $\begin{array}{l}\text { Nivel de conocimiento de } \\
\text { infraestructura de manejo de } \\
\text { agua pluvial }\end{array}$ & Capacidad de infiltración & \multirow[t]{2}{*}{ Costo de construcción } & \multirow[t]{2}{*}{$\begin{array}{c}\text { Incremento de áreas verdes } \\
\text { urbanas }\end{array}$} \\
\hline Participación social & Adaptabilidad & & \\
\hline $\begin{array}{l}\text { Interés en el manejo de agua } \\
\text { pluvial }\end{array}$ & Funcionabilidad & \multirow{2}{*}{$\begin{array}{l}\text { Reducción de costos por } \\
\text { reutilización de materiales }\end{array}$} & \multirow{2}{*}{$\begin{array}{c}\text { Grado de reducción de la } \\
\text { contaminación de suelo y } \\
\text { agua subterránea }\end{array}$} \\
\hline $\begin{array}{c}\text { Interés de inversión en } \\
\text { infraestructura para drenaje } \\
\text { pluvial }\end{array}$ & Reutilización de materiales & & \\
\hline
\end{tabular}

Fuente: elaboración propia.

A cada uno de los indicadores se le asignó un valor de 0 a 4, de acuerdo con la Tabla 1. La ponderación óptima se determinó con la ecuación 5; la ponderación obtenida para el proyecto se estableció con la ecuación 6 y el porcentaje de viabilidad para cada componente se obtuvo con la ecuación 7 (Cuadro 5). 
Cuadro 5. Puntaje y ponderación óptima, puntaje y ponderación obtenida y porcentaje de viabilidad para las componentes social, técnica, económica y ambiental

\begin{tabular}{|l|c|c|c|c|c|}
\hline Componente & $\begin{array}{c}\text { Puntaje } \\
\text { óptimo }\end{array}$ & $\begin{array}{c}\text { Ponderación } \\
\text { óptima (\%) }\end{array}$ & $\begin{array}{c}\text { Puntaje } \\
\text { obtenido }\end{array}$ & $\begin{array}{c}\text { Ponderación } \\
\text { obtenida (\%) }\end{array}$ & \% de viabilidad \\
\hline Social & 24 & 33.33 & 16 & 22.22 & $\mathbf{6 6 . 6 7}$ \\
\hline Técnica & 24 & 33.33 & 20 & 27.78 & $\mathbf{8 3 . 3 3}$ \\
\hline Económica & 12 & 16.67 & 9 & 12.5 & $\mathbf{7 5}$ \\
\hline Ambiental & 12 & 16.67 & 11 & $\mathbf{7 7 . 7 8}$ & \\
\hline Total & $\mathbf{7 2}$ & $\mathbf{1 0 0}$ & $\mathbf{5 6}$ & & \\
\hline
\end{tabular}

Fuente: elaboración propia.

Los porcentajes de viabilidad obtenidos para cada componente diagnosticada muestran que el proyecto cuenta con viabilidad social, técnica, económica y ambiental, al presentar valores por arriba del $66 \%$. En lo que concierne a la ponderación total obtenida para el proyecto, ésta tuvo un valor de $77.78 \%$, por lo que se puede considerar que el proyecto es viable en áreas con las características técnicas similares a las colonias Carolino Anaya y Progreso Macuiltépetl. Con relación a estos resultados, Joksimovic y Alam (2014) resaltan que las zanjas y pozos de infiltración son las soluciones más rentables para la reducción de escurrimiento superficial y encharcamientos generados por las lluvias, lo cual se confirma en el presente estudio.

\section{Conclusiones}

Técnicamente, la implementacion de pozos de infiltración en la zona urbana de Xalapa, dadas las características geotécnicas de suelo estable, resulta viable. Desde el punto de vista social es también viable, ya que existe la disposición por parte de los habitantes de construir sus pozos de infiltración en virtud de los beneficios que ofrece. En cuanto a lo económico, igualmente hay condiciones suficientes para su implementación, ya que son de bajo costo (\$1 153.38), y por último, en términos ambientales resulta ser también una opción adecuada, ya que reduciría el escurrimiento del agua pluvial en un $25 \%$ en el área urbana de Xalapa donde se le implemente, disminuyendo encharcamientos e inundaciones.

Las características técnicas como tipo de suelo, geotecnia y capacidad de infiltración son prioritarias para el uso de drenaje pluvial alternativo en viviendas de Xalapa. Si bien los pozos de infiltración a nivel vivienda ya se emplean en otros países, y en México son considerados en algunos proyectos de planificación urbana como parte de la gestión del agua pluvial, son una 
tecnología para el manejo del agua de lluvia amigable con el ambiente que podría ser adoptada a mayor escala, como complemento del sistema de drenaje convencional.

\section{Agradecimientos}

Los autores agradecen al CONACyT por la beca de posgrado número 279412 otorgada al primer autor para realizar este trabajo.

\section{Referencias}

Ando, Amy y Freitas, Luiz (2011). "Consumer Demand for Green Stormwater Management Technology in an Urban Setting: The Case of Chicago Rain Barrels". Water Resources Research, 47(12), pp. 1-11. doi: $\underline{10.1029 / 2011 W R 011070}$

Ashley, Richard; Lundy, Lian; Ward, Sarah; Shaffer, Paul; Walker, Louise; Morgan, Celeste; Saul, Adrian; Wong, Tony, y Moore, Sarah (2013). "Water-Sensitive Urban Design: Opportunities in the UK". Proceedings of the Institution of Civil Engineers: Municipal Enginner, 166(2), pp. 6576. doi: $\underline{10.1680 / m u e n .12 .00046}$

Audefroy, Joel y Padilla, Raymundo (2018). Desastres asociados a fenómenos hidrometeorológicos. Jiutepec, México: IMTA, 260 pp.

Autixier, Laurène; Mailhot, Alain; Bolduc, Samuel; Madoux-Humery, AnneSophie; Galarneau, Martine; Prévost, Michèle, y Dorner, Sarah (2014). "Evaluating Rain Gardens as a Method to Reduce the Impact of Sewer Overflows in Sources of Drinking Water". Science of the Total Environment, 499, pp. 238-247. doi: 10.1016/j.scitotenv.2014.08.030

Azzout, Yolande; Barraud, Sylvie, y Cres, Francois-Noel (1994). Techniques alternatives en assainissement pluvial: choix, conception, réalisation et entretien. París, Francia: Technique et Documentation Lavoisier, 371 pp.

Brito-Castillo, Luis y Pedrozo-Acuña, Adrián (2015). "Inundaciones, heladas e incendios forestales". En Teresa Cavazos (ed.), Conviviendo con la naturaleza: El problema de los desastres asociados a fenómenos hidrometeorológicos y climáticos en México. Tijuana, México: Ediciones ILCSA, pp. 78-119.

Bouarafa, Sofia; Lassabatere, Laurent; Lipeme-Kouyi, Gislain, y AnguloJaramillo, Rafael (2019). "Hydrodynamic Characterization of 
Sustainable Urban Drainage Systems (SuDS) by Using Beerkan Infiltration Experiments", Water, 11(4), pp. 1-17. doi: 10.3390/w1104 $\underline{0660}$

Campos-Aranda, Daniel (2010). Introducción a la hidrología urbana. San Luis Potosí, México: Printego, 270 pp.

Chang, Heejun; Franczyk, Jon, y Kim, Changhwan (2009). "What is Responsible for Increasing Flood Risk? The Case of Gangwon Province, Korea”. Natural Hazards, 48(3), pp. 339-354. doi: 10.1007/s11069-008$\underline{9266-\mathrm{y}}$

CIDECALLI (2001). Manual sobre sistemas de captación y aprovechamiento del agua de lluvia para uso doméstico y consumo humano. Montecillo, México: Colegio de Posgraduados, $101 \mathrm{pp}$.

CONAGUA (2019). Manual de agua potable, alcantarillado y saneamiento: Drenaje pluvial urbano. México D.F.: SEMARNAT, 454 pp.

Ellis, John; Lundy, Lian, y Revitt, Michael (2016). "The Regulatory Framework for Urban Stormwater Flood Management in England and Wales: Challenges for the Implementation of Sustainable Drainage Systems", Novatech, pp. 1-8. Recuperado de http://documents.irevues. inist.fr/bitstream/handle/2042/60376/3A91-083LUN.pdf

Fanelli, Rosemary; Prestegaard, Karen, y Palmer, Margaret (2017). "Evaluation of Infiltration-Based Stormwater Management to Restore Hydrological Processes in Urban Headwater Streams". Hydrological Processes, 31(19), pp. 3306-3319. doi:/10.1002/hyp.11266

García, Eduardo (2011). "Control de escorrentías urbanas mediante pavimentos permeables: aplicación en climas mediterráneos" (Tesis de maestría en Ingeniería Hidráulica y Medio Ambiente). Recuperado de https://riunet.upv.es/bitstream/handle/10251/15383/TFM_EGH.pdf?seq uence $=1 \&$ isAllowed $=y$

García, Enriqueta (2004). Modificaciones al sistema de clasificación climática de Köppen (Para adaptarlo a las condiciones de la República Mexicana). México D.F.: Instituto Nacional de Geografía-UNAM, 90 pp.

Gutiérrez, Alejandra (2017). "Gestión sustentable del agua en la vivienda de interés social en el Área Metropolitana de Guadalajara (AMG). Escenarios deseables a partir de la modelación sustentable de la infraestructura hídrica”. Vivienda y comunidades sustentables, 1(2), pp. 39-50. doi: 10.32870/rvcs.v0i2.23 
Huesca, Daniel (2009). "Evaluación comparativa de la respuesta de sitio en las distintas unidades geológicas-geotécnicas en la zona conurbada Xalapa” (Tesis de Ingenieria Civil). Xalapa, Veracruz, México: Universidad Veracruzana, 132 pp.

Imbe, Masahiro; Takano, Noboru, y Nakashima, Noriyuki (1995). "Proposal for the Standardized Design of Infiltration Techniques of Rainstorm Water in Japan”. Novatech, 95, pp. 645-648.

INEGI (2014). “Marco Geoestadístico Municipal”, versión 3.2. Recuperado de https://www.inegi.org.mx/app/biblioteca/ficha.html?upc $=702825004386$

Jaume, Arturo (2016). "Gestión de las aguas pluviales en entornos urbanos mediante técnicas de Drenaje Sostenible". Journal of Engineering and Technology, 5(2), pp. 26-40. doi: 10.22507/jet.v5n2a1

Joksimovic, Darko y Alam, Zakia (2014). "Cost Efficiency of Low Impact Development (LID) Stormwater Management Practices". Procedia Engineering, 89, pp. 734-741. doi: 10.1016/j.proeng.2014.11.501

Katsifarakis, Konstantinos; Vafeiadis, Marios, y Theodossiou, Nicolaos (2015). "Sustainable Drainage and Urban Landscape Upgrading Using Rain Gardens. Site Selection in Thessaloniki, Greece". Agriculture and Agricultural Science Procedia, 4, pp. 338-347. doi: 10.1016/j.aaspro.2015.03.038

Kogan, Jorge y Bondorevsky, Diego (2016). "La infraestructura en el desarrollo de América Latina”. Economía y desarrollo, 156(1), pp. 168186. Recuperado de http://scielo.sld.cu/pdf/eyd/v156n1/eyd12116.pdf

Lemoine, Richard (2012). "Cambios en la cobertura vegetal de la ciudad de Xalapa-Enríquez, Veracruz y zonas circundantes entre 1950 y 2010" (Tesis de Licenciatura en Biología). Xalapa, Veracruz, México: Universidad Veracruzana, $44 \mathrm{pp}$.

Lizárraga-Mendiola, Liliana; Vázquez-Rodríguez, Gabriela; Blanco-Piñón, Alberto; Rangel-Martínez, Yamile, y González-Sandoval, María (2015). "Estimating the Rainwater Potential per Household in an Urban Area: Case Study in Central Mexico". Water, 7(9), pp. 4622-4637. doi: 10.3390/w7094622

Loperfido, John; Noe, Gregory; Jarnagin, Taylor, y Hogan, Dianna (2014). "Effects of Distributed and Centralized Stormwater Best Management Practices and Land Cover on Urban Stream Hydrology at the Catchment Scale”. Journal Hydrology, 519, pp. 2584-2595. doi: 10.1016/j.jhydrol. $\underline{2014.07 .007}$ 
Massolo, Laura (2015). Introducción a las herramientas de gestión ambiental. Buenos Aires, Argentina: Universidad Nacional de La Plata, $195 \mathrm{pp}$.

Mikkelsen, Peter Steen; Häfliger, Martin; Ochs, Michael; Tjell, Jens Christian; Jacobsen, Peter, y Boller, Markus (1996). "Experimental Assessment of Soil and Groundwater Contamination from Two Old Infiltration Systems for Road Run-Off in Switzerland". Science of the Total Environment, 189, pp. 341-347. doi: 10.1016/0048-9697(96)05229-1

MINVU-PUC (1996). Técnicas alternativas para soluciones de agua de lluvias en sectores urbanos. Guía de diseño. Recuperado de http://minvuhistorico.minvu.cl/opensite_det_20070317115825.aspx

Moguel, Ana (2011). "Riesgos por inundaciones en la Ciudad de Xalapa, Ver." (Tesis de Licenciatura en Ciencias Atmosféricas). Xalapa, Veracruz, México: Universidad Veracruzana, $49 \mathrm{pp}$.

Mulder, Karel (2007). Desarrollo sostenible para ingenieros. Barcelona, España: Ediciones UPC, 240 pp.

Ossa-Moreno, Juan; Smith, Karla, y Mijic, Ana (2017). "Economic Analysis of Wider Benefits to Facilitate SuDS Uptake in London, UK". Sustainable Cities and Society, 28, pp. 411-419. doi: 10.1016/j.scs.2016.10.002

Pacheco, Verónica; Carrera, Patricia, y Almeida, Katalina (2011). "Propuesta metodológica para la evaluación de la factibilidad de proyectos de turismo comunitario: Caso de estudio: Comunidades Huaorani, Achuar y Shiwiar de la Amazonia Ecuatoriana”. Gestión turística, 15, pp. 21-46. Recuperado de https://www.redalyc.org/pdf/2233/223318904002.pdf

Papafotiou, Epaminondas y Katsifarakis, Konstantinos (2015). "Ecological Rainwater Management in Urban Areas. Preliminary Considerations for the City of Corinth, Greece". Agriculture and Agricultural Science Procedia, 4, pp. 383-391. doi: 10.1016/j.aaspro.2015.03.043

Peña, Leticia (2014). "Propuesta de rehabilitación urbana para la colonia tarahumara en Ciudad Cuauhtémoc, Chihuahua, México". Hábitat sustentable, 4(2), pp. 64-73. Recuperado de http://revistas.ubiobio.cl/ index.php/RHS/article/view/451

Perales-Momparler, Sara y Andrés-Doménech, Ignacio (2008). "Los sistemas urbanos de drenaje sostenible: una alternativa a la gestión del agua de lluvia”. Revista técnica de medio ambiente. C\&M publicaciones, 124, pp. 92-104. Recuperado de http://ovacen.com/wp-content/ uploads/2015/05/ gestion-del-agua-en-el-planeamiento.pdf 
Perales-Momparler, Sara; Hernández-Crespo, Carmen; Vallés-Morán, Francisco; Martín, Miguel; Andrés-Doménech, Ignacio; Álvarez, Joaquín, y Jefferies, Chris (2014). "SUDS efficiency during the Start-Up period under mediterranean climatic conditions". Clean-soil, air, water, 42(2), pp. 178-186. doi: 10.1002/clen.201300164

Pereyra-Díaz, Domitilo; Ochoa-Martínez, Carolina, y Pérez-Sesma, José Antonio (2005). "Ecuaciones de lluvia intensa generalizada para obtener precipitaciones máximas de corta duración”. Geos, 25(2), pp. 340-351. Recuperado de https://www.ugm.org.mx/publicaciones/geos/pdf/geos052/a.pdf

Qin, Hua-peng; Li, Zhuo-xi, y Fu, Guangtao (2013). "The Effects of Low Impact Development on Urban Flooding under Different Rainfall Characteristics". Journal of Environmental Management, 129, pp. 577585. doi: 10.1016/j.jenvman.2013.08.026

Rodríguez-Eugenio, Natalia; McLaughlin, Michael, y Pennock, Daniel (2019). La contaminación del suelo: una realidad oculta. Roma, Italia: FAO, 130 pp.

Rojas, Franz; Peñaherrera, Fernando; Orellana, Carlos; Castañeda, Helena; Armijos, Leonardo; Burbano, Luis; Morales, Antonio; Rodrigues, Paulo; Real, Carlota; Rispo, Andrea; Valverde, Osvaldo; Alonso, Agustín, y Bianchi, Fabiana (2019). Estrategia del agua 2019-2022. Buenos Aires, Argentina: CAF, $71 \mathrm{pp}$.

Romero-Guzmán, Liliana; De Hoyos-Martínez, Jesus, y Romero-Guzmán, Elizabeth (2018). "Ciudades sustentables a partir de un paradigma hídrico emergente, caso de estudio: Toluca, México". Legado de arquitectura y diseño, 13(23), 107-118. Recuperado de http://www.redalyc.org/articulo.oa?id=477954382032

Sánchez-Rodríguez, Roberto y Cavazos, Tereza (2015). “Amenazas naturales, sociedad y desastres". En Teresa Cavazos (ed.), Conviviendo con la naturaleza: El problema de los desastres asociados a fenómenos hidrometeorológicos y climáticos en México. Tijuana, México: Ediciones ILCSA, pp.1-45.

Serrano, Sebastián (2014). "Aprovechar el agua de lluvia. Doble solución”. Impluvium, (1), pp. 23-27. Recuperado de http://www.agua.unam.mx/ assets/pdfs/impluvium/numero01.pdf

SMN (s/f) "Normales climatológicas por estados_Veracruz". Recuperado de https://smn.conagua.gob.mx/es/informacion-climatologica-porestado?estado=ver (consultado el 2 de diciembre de 2019). 
Spiegel, Murray y Stephens, Larry (2009). Estadística. México, D.F.: McGraw-Hill, 577 pp.

Stovin, Virginia; Moore, Sara; Wall, Matthew, y Ashley, Richard (2013). "The Potential to Retrofit Sustainable Drainage Systems to Address Combined Sewer Overflow Discharges in the Thames Tideway Catchment". Water and Environment Journal, 27(2), pp. 216-228. doi: 10.1111/j.1747-6593.2012.00353.x

Suárez, Antonio; Camarena, Pedro; Herrera, Ismael, y Lot, Antonio (2008). Infraestructura verde y corredores ecológicos de los pedregales: ecología urbana del sur de la Ciudad de México. Recuperado de http://centro.paot.org.mx/documentos/unam/infraestructura_verde.pdf

Valderrama, Alisa y Levine, Larry (2012). Financing Stormwater Retrofits in Philadelphia and beyond. Recuperado de http://cpicfinance.com/wpcontent/uploads/2017/03/StormwaterFinancing-report.pdf

VanWoert, Nicholaus; Rowe, Bradley; Andresen, Jeffrey; Rugh, Clayton; Fernandez, Thomas, y Xiao, Lan (2005). "Green Roof Stormwater Retention: Effects of Roof Surface, Slope, and Media Depth". Journal of Environmental Quality, 34, pp. 1036-1044. doi: 10.2134/jeq2004.0364

Walsh, Christopher; Fletcher, Tim, y Burns, Matthew (2012). "Urban Stormwater Runoff: A New Class of Environmental Flow Problem”. Plos One, 7(10). pp. e45814. doi: 10.1371/journal.pone.0045814

Walsh, Thomas; Pomeroy, Christine, y Burian, Steven (2014). "Hydrologic Modeling Analysis of a Passive, Residential Rainwater Harvesting Program in an Urbanized, Semi-Arid Watershed”. Journal of Hydrology, 508, pp. 240-253. doi: 10.1016/j.jhydrol.2013.10.038

Woods-Ballard, Bridget; Wilson Steve; Udele-Clark, Helen; Illman, Sue; Ashley, Richard, y Kellagher, Richard (2015). The SUDS Manual. Londres, Reino Unido: CIRIA C753, 937 pp.

Editor asociado: Juan Carlos Pérez Jiménez

Recibido: 15 de abril de 2020 Aceptado: 25 de agosto de 2020 